\title{
Genetic mutation of familial dilated cardiomyopathy based on next-generation semiconductor sequencing
}

\author{
XIN-FU LIN ${ }^{1,2^{*}}$, JIE-WEI LUO ${ }^{1,3^{*}}$, GUI LIU ${ }^{3 *}$, YAO-BIN ZHU ${ }^{4}$, ZHAO JIN ${ }^{1}$ and XING LIN ${ }^{1,5}$ \\ ${ }^{1}$ Provincial Clinical Medical College, Fujian Medical University; Departments of ${ }^{2}$ Paediatrics and \\ ${ }^{3}$ Traditional Chinese Medicine, Fujian Provincial Hospital, Fuzhou, Fujian 350001; ${ }^{4}$ Department of \\ Traditional Chinese Medicine, The First Affiliated Hospital, Fujian Medical University, Fuzhou, Fujian 350005; \\ ${ }^{5}$ Department of Thoracic Surgery, Fujian Provincial Hospital, Fuzhou, Fujian 350001, P.R. China
}

Received January 11, 2018; Accepted August 2, 2018

DOI: $10.3892 / \mathrm{mmr} .2018 .9455$

\begin{abstract}
Dilated cardiomyopathy (DCM) is a complex myocardial disease of multifactorial etiologies, including enlarged cardiac chambers and contractile dysfunction. It has been suggested that the inheritance of DCM-associated mutations predominates its onset. Therefore, the present study investigated the pathogenesis of DCM via pedigree analysis and genetic diagnosis by massive whole-exome screening, and targeted exon capture. To study the familial gene-phenotype association, the exon and splice sites of 325 hereditary disease-associated genes in the proband with familial dilated cardiomyopathy (FDC), including 61 cardiac diseaseassociated genes, such as the lamins $\mathrm{A} / \mathrm{C}(L M N A)$, were analyzed by ultra-high multiplex polymerase chain reaction and the Ion AmpliSeq $^{\mathrm{TM}}$ Inherited Disease Panel. The present study also conducted Sanger DNA Sequencing for family members with global minor allele frequencies $<1 \%$ to verify potential pathogenic mutation sites. A total of three rare missense mutations were detected, including heterozygous c. $244 \mathrm{G}>\mathrm{A}$ in $L M N A$, c. $546 \mathrm{C}>\mathrm{G}$ in potassium voltage-gated channel subfamily KQT (KCNQ4) and c.1276G $>\mathrm{A}$ in EYA transcriptional coactivator and phosphatase 1 (EYAl), indicating a glutamic acid to lysine
\end{abstract}

Correspondence to: Dr Jie-Wei Luo, Department of Traditional Chinese Medicine, Fujian Provincial Hospital, 134 Dongjie, Fuzhou, Fujian 350001, P.R. China

E-mail: docluo0421@aliyun.com

${ }^{*}$ Contributed equally

Abbreviations: DCM, dilated cardiomyopathy; FDC, familial dilated cardiomyopathy; LMNA, lamins A/C; MAF, global minor allele frequency; EYA1, EYA transcriptional coactivator and phosphatase 1; LV, left ventricular; AP4, apical four chamber view; RVD, right ventricular diameter; LVEF, left ventricular ejection fraction

Key words: lamins A/C, potassium voltage-gated channel subfamily KQT, EYA transcriptional coactivator and phosphatase 1, mutations, familial dilated cardiomyopathy, atrioventricular block substitution at amino acid 82 (p.E82K) in $L M N A$, a p.F182L in KCNQ4 (a mutation associated with pathogenic deafness) and p.G426S in EYAl (associated with Branchiootorenal syndrome 1 and Branchiootic syndrome 1 pathogenesis). In the present study, a carrier with slight hearing impairment was detected in the family analyzed; however, no patients with deafness or branchiootorenal syndrome were observed. LMNA p.E82K revealed SIFT and PolyPhen-2 scores of 0 and 1 , respectively. In the second generation, 3 patients with DCM underwent permanent pacemaker implantation due to sick sinus syndrome, atrioventricular block and unstable cardiac electrophysiology. The present study suggested that LMNA p.E82K may contribute to the pathogenesis of FDC and concomitant atrioventricular block. At present, only three families with DCM resulting from similar mutations have been reported. The present study demonstrated the strong pathogenic effects of LMNA p.E82K on DCM.

\section{Introduction}

Familial dilated cardiomyopathy (FDC) has been identified in at least two closely related patients with dilated cardiomyopathy (DCM) meeting the criteria for idiopathic dilated cardiomyopathy (IDC). In addition, DCM has a complex pathogenesis. Genetic screening methods have demonstrated that common variants in numerous genes are present in severe DCM cases, indicating that patients with DCM have complex multi-variant or oligogenic genetic backgrounds (1-3). It has been suggested that up to $50 \%$ of idiopathic DCM cases may be attributed to genetic mutations (4). It may therefore be suggested that family heredity has a major role in the pathogenesis of DCM. Novel diagnosis of IDC has indicated FDC in first-degree family members at a minimum rate of $20-35 \%$ via clinical screening. Diverse gene ontogeny, or more specifically, point mutations in 31 autosomal and $2 \mathrm{X}$-linked genes, have been reported to be associated with the incidence of FDC; however, such factors account for 30-35\% of the genetic causes of FDC (1). The majority of patients with FDC exhibit a dominant inheritance pattern; however, a small minority of patients with DCM possessing other inheritance patterns have been reported, including autosomal recessive, X-linked and mitochondrial modes of inheritance (1). Hence, a published 
clinical guideline strongly suggests that families with a history of cardiomyopathy should undergo genetic testing and screening in the clinic (4). These genes and loci have been identified in numerous families, coding for a variety of cardiomyocyte proteins of the cytoskeleton, nuclear membrane, the sarcomere, ion channels and the sarcolemma. The proportion of protein-coding genes with pathogenic mutations resulting in FDC include that of Titin (15-27\%), lamins A/C (LMNA; $6 \%), \beta$-myosin heavy chain $(4.20 \%)$, myopalladin $(3.50 \%)$ and cardiac troponin $\mathrm{T}(2.90 \%)(5)$. Titin is a large cytoskeletal protein of the muscle, which regulates muscle extensibility and fiber elasticity; lamins A/C stabilize the nuclear membrane and provides mechanical support. Furthermore, $\beta$-myosin heavy chain, myopalladin and cardiac troponin $\mathrm{T}$ are sarcoma proteins, all of which serve roles in regulating muscle contraction. Phospholamban, encoded by $P L N$, is a sarcoplasmic reticulum protein that can inhibit the sarcoplasmic reticulum $\mathrm{Ca}^{2+}$-ATPase pump and regulate muscle function. As many as $6 \%$ of patients with DCM possess mutations in LMNA, among of which almost 30-33\% of these patients with DCM suffered from conduction system disorders $(6,7)$. In addition, carriers of LMNA and PLN mutations were more likely to develop malignant ventricular arrhythmia and end-stage heart failure (8).

LMNA is located on chromosome 1q21-22 and is an autosomal dominant gene constituting 12 exons, and encodes two isomers, lamin A and lamin C. Lamins A/C are transcript shears of $L M N A$ mRNA that are selectively translated and contain 566 similar residues proximal to the $\mathrm{N}$-terminus. Selective cleavage within exon 10 results in two different mRNAs encoding prelamin $\mathrm{A}$ and lamin C. Prelamin A constitutes 664 residues, the precursor protein of lamin; its C-terminus is modified by farnesylation. Following the loss of 3 amino acids, C-terminal methylation and cleavage of the internal soluble protein occur, a synthetic mature version of lamin A is produced, constituting 646 amino acids, which is then inserted into the nuclear lamina. Lamins $\mathrm{A} / \mathrm{C}$ contain the 'rod' functional region of an $\alpha 2$ helix structure comprising 360 amino acid residues, which is composed of 7 repeating hydrophobic amino acid sequences; the components on each end are known as the N-terminal 'head' and the C-terminal 'tail'.

Exon 1 is responsible for encoding the first region of the $\mathrm{N}$-terminal head; exons 2-6 encode the remaining central rod-shaped region, whereas exons 7-9 encode the C-terminal region, including the nuclear localization signal region and the binding domain for nuclear intermediate filament protein to DNA $(9,10)$. Cell nuclear lamins that localize to the inner nuclear membrane consist of lamins $\mathrm{A} / \mathrm{C}$, and $\mathrm{B}$, the latter is encoded by the $L M N B$ gene, and provides mechanical support to maintain the integrity of the nuclear membrane (11). In addition, lamins $\mathrm{A} / \mathrm{C}$ have been reported crucial for the function of a chromosome anchor site, periodic nuclear degradation and assembly, as well as the regulation cellular proliferation, differentiation and apoptosis, cellular signal transduction, chromosome segregation, gene regulation and DNA repair (12).

\section{Materials and methods}

Clinical data of the proband and familial members. The present study investigated the clinical data of the proband and familial members (Table I; Fig. 1). The proband (II-6) was a 54-year-old female whose main symptoms were recurrent chest congestion and pain with shortness of breath for $>1$ year. The patient was admitted to Fujian Provincial Hospital (Fuzhou, China) in March 2008. The results of physical examination revealed a pulse of $>60$ beats per minute (BPM), blood pressure of 137/67 mmHg, a lack of jugular vein distension and clear breathing without rales. Furthermore, the relative cardiac dullness expanded to both sides, the heart rate was 60 BPM and heart rhythm was uniform; a 3/6 grade systolic murmur in accordance with the Levine classification method (13) at the second intercostal space of the left sternal border was reported and no lower extremity edema was observed. Repeated electrocardiogram examinations indicated that the patient had frequent ventricular premature beats (bigeminy), poor $\mathrm{R}$ wave progression on the $\mathrm{V}_{1}-\mathrm{V}_{4}$ leads, sinus bradycardia, first-degree atrioventricular block, notable U waves, a low ST segment on part of the leads and T-wave inversion. Echocardiographic screening revealed that from the perspective of the parasternal long-axis view of the left ventricle (LV), the left atrial diameter was $3.76 \mathrm{~cm}$ and the LV end-diastolic diameter was $5.68 \mathrm{~cm}$, the right atrial diameter (apical four-chamber view, AP4) was $4.18 \mathrm{~cm}$, and the right ventricular diameter (RVD; AP4) was $4.06 \mathrm{~cm}$. Additionally, the LV ejection fraction (LVEF) was $35 \%$ and the LV fractional shortening was $23 \%$, which suggested decreased overall motion of the left ventricular wall. Furthermore, a reduced LVEF, LV enlargement with moderate mitral valve regurgitation, and right atrium and RV enlargement with moderate tricuspid regurgitation were observed. There was no previous history of heart disease due to the enlargement of heart, smoking and alcohol consumption of the proband.

Myocardial perfusion imaging demonstrated that the left ventricular cavity was slightly enlarged, the thickness of the ventricular wall was uneven, the perfusion of blood to the LV was decreased, and the anterior wall was close to the apex and the posterior wall. This was consistent with the phenotypic characteristics for DCM (14), as determined by the New York Heart Association Standard Committee revised cardiac function classification (15). A Holter monitor indicated a junctional rhythm with an average heartrate of 44 BPM. The longest R-R interval was $1.69 \mathrm{sec}$; there were 2,431 instances where the R-R interval was longer than $1.6 \mathrm{sec}$. An occasional ventricular premature beat ( 22 beats/24 h, one paired beat $/ 24 \mathrm{~h}$, dual-source), paroxysmal ventricular tachycardia (2 times/24 h) and a ST-T change were also observed. Subsequently, the patient underwent permanent pacemaker implantation. After 6.5 years following hospital admission, the patient had permanent resynchronization pacemaker replacement surgery again as the battery of the pacemaker had worn. After 7 months following the operation, the Holter monitor revealed paroxysmal atrial fibrillation with the pacemaker where the fastest ventricular rate was as high as 86 BPM (atrial fibrillation) and the lowest ventricular rate was as low as 60 BPM (pacemaker heart rate). The pacemaker functioned as a dual-chamber model with a frequency of 60 BPM and a ventricular premature beat frequency of 96 beats per $24 \mathrm{~h}$.

In the second generation, a total of 3 patients with DCM (II-5, -6 and -7) underwent permanent pacemaker implantation due to sick sinus syndrome and unstable cardiac 


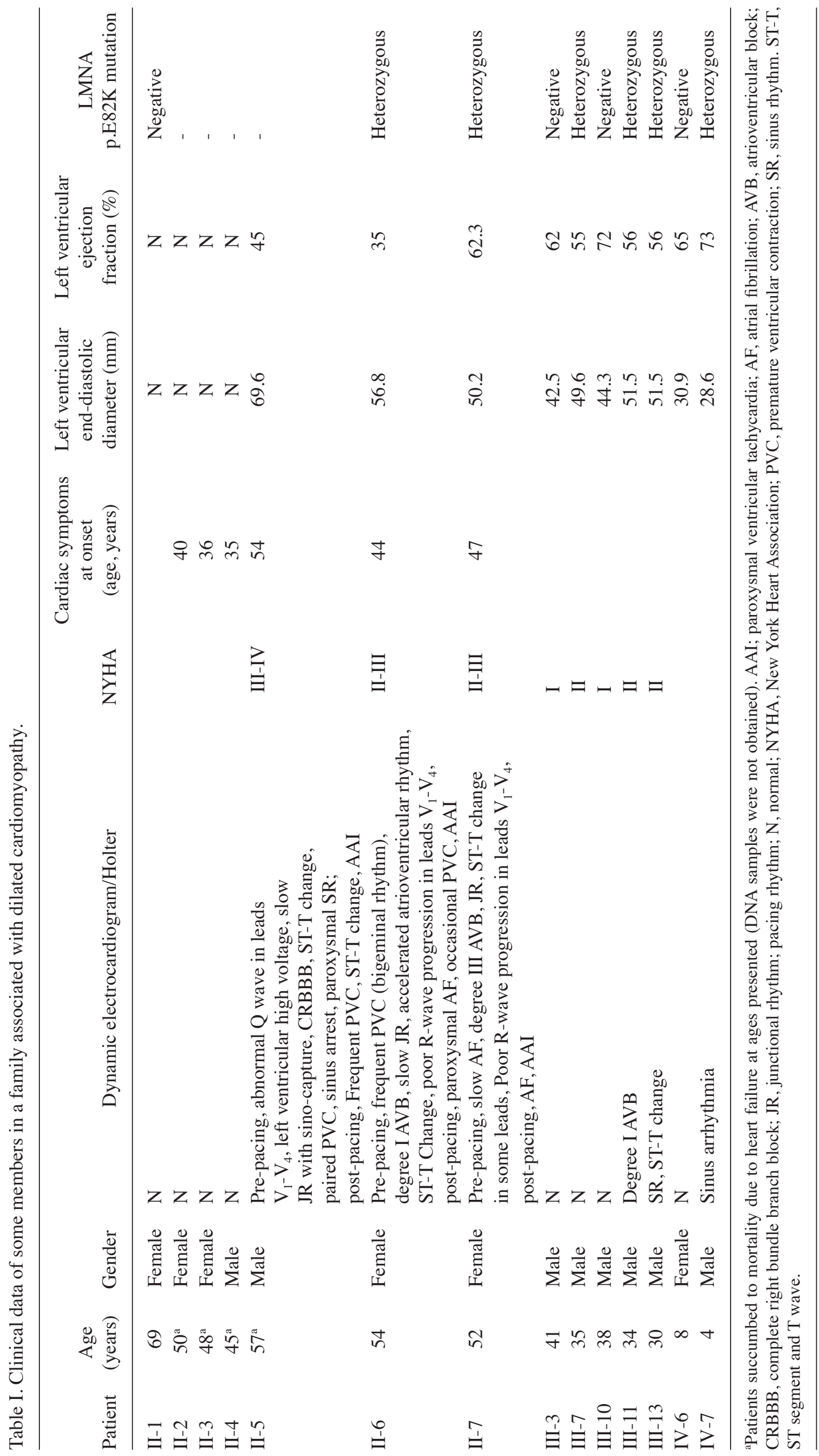




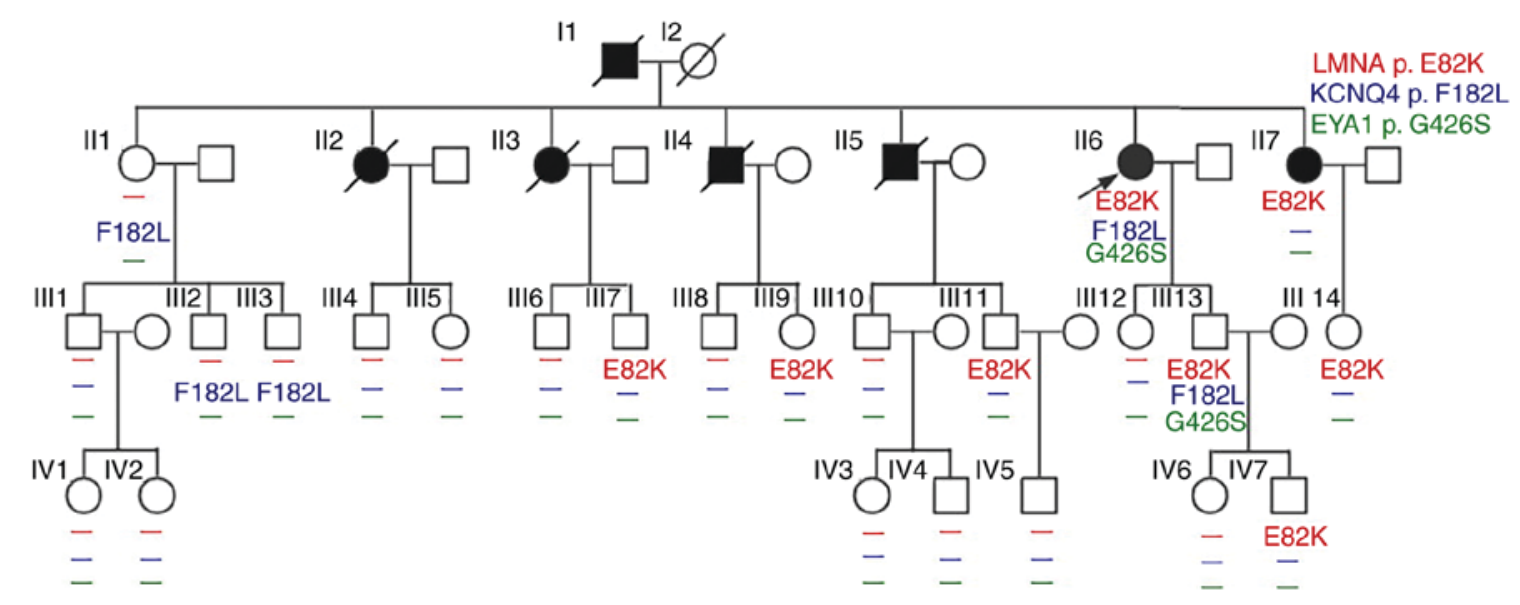

Figure 1. Pedigree chart and genotypes of the family with dilated cardiomyopathy. Males and females are indicated by squares and circles, respectively, and members presenting with dilated cardiomyopathy are filled in black. Arrow indicates the proband. The diagonal line indicates deceased individuals. LMNA, lamins A/C; KCNQ4, potassium voltage-gated channel subfamily KQT; EYA1, EYA transcriptional coactivator and phosphatase 1.

electrophysiology. Additionally, two sisters (II-2 and II-3) and an elder brother (II-4) of the proband (II-6) in this family succumbed to mortality due to heart disease or heart failure at 40-50-years-old. An additional family member (III-3) revealed a slight decline in hearing ability of the left ear via an audiometry examination; however, the hearing of II-6, II-1, III-2 and III-13, and the right ear hearing of III-3 was normal. Pedigree analysis revealed no family members with auricle or external auditory canal deformities, preauricular fistula, branchial cleft anomalies, renal dysplasia, or any other symptoms.

DNA extraction, capture of target gene and library preparation. Peripheral blood samples $(2 \mathrm{ml})$ obtained from the forearm were used to extract genomic DNA using the QIAamp DNA Blood Mini kit (Qiagen GmbH, Hilden, Germany). The concentration and purity of DNA were measured with a Nanodrop 1000 spectrophotometer (NanoDrop Technologies; Thermo Fisher Scientific, Inc., Pittsburgh, PA, USA) according to the manufacturer's protocol.

Amplification of target genes was performed using the Ion Ampliseq ${ }^{\mathrm{TM}}$ Inherited Disease Panel (Thermo Fisher Scientific, Inc.) as mentioned below. In order to arrange the genes according to their sequences, a next-generation sequencing (NGS) library was prepared. A total of $20 \mathrm{ng}$ gDNA obtained from each sample was quantified using a Qubit 2.0 fluorometer (Invitrogen; Thermo Fisher Scientific, Inc.), and multiplex PCR amplification using each of the two primer-pools was subsequently performed in accordance with the manufacturer's instructions. Following the mixing of the resultant amplicons from the two primer-pools, the amplicons were ligated to barcodes and Ion Torrent adapters (Thermo Fisher Scientific, Inc.). Agencourt AMPure XP beads (Beckman Coulter, Inc., Brea, CA, USA) were then utilized for purification of the amplified libraries using an Ion AmpliSeq Library kit 2.0, which was performed in accordance with the manufacturer's instructions (Thermo Fisher Scientific, Inc.) (16).

Personal Genome Machine (PGM) sequencing and variant calling. Ion 318 chips were employed with the Ion PGM system (Thermo Fisher Scientific, Inc.). Ion Torrent Suite Software v3.6.2 (Thermo Fisher Scientific, Inc.) was used to i) collect data of Ion Torrent reads, ii) determine trim adapter sequences (clean data), iii) align clean data to the hg19 human reference genome, iv) analyze coverage of target sequence and v) variant calling (16). Furthermore, in accordance with the quality score system of Ion Torrent, the software scores for the quality of the reads were computed via assignment of the Q17 and Q20 scores. Following this, primers of overlapping amplicons covering the coding sequence region and flanking sequences of each target gene were designed by Ion AmpliSeq ${ }^{\mathrm{TM}}$ Ready-to-Use custom designer platform (v7.0.2; https://www.ampliseq.com/protected/dashboard. action). The reaction system and conditions of polymerase chain reaction (PCR) were performed using the AmpliSeq ${ }^{\mathrm{TM}}$ Inherited Disease Panel (Thermo Fisher Scientific, Inc.) in accordance with the manufacturer's instructions. Multiple PCR amplifications were performed for 325 genes associated with genetic disease (61 heart disease-associated genes, such as $L M N A$ ) and the region near the splice sites. Mutated polymorphic sites that had a global minor allele frequency (MAF) of $\leq 1 \%$ were screened, which was calculated from the population frequency information regarding dbSNP obtained from the 1000 genomes project (17). The identified mutation points were compared via Ion reporter 5.0 (https://ionreporter.thermofisher.com/ir/) against thefollowing databases: NCBI dbSNP database (https://www.ncbi.nlm.nih. gov/snp/), the 1000-Genome project database (http://www. internationalgenome.org/category/dbsnp/), UCSC common SNP database (https://genome.ucsc.edu/goldenpath/gbdDescriptionsOld.html)and the 5000Exmoes database in Exome Sequencing Project (https://ionreporter.thermofisher.com/ir/). The SIFT (SIFT v4.0.3; http://provean.jcvi. org/index.php) and PolyPhen-2 (Polymorphism Phenotyping v2; http://genetics.bwh.harvard.edu/pph2/) were used to predict missense mutation protein function, and Phenotyping v2 was also used to determine species conserved sequences and to perform protein homology analysis. LMNA GeneView (https://www.uniprot.org/uniprot/P02545\#structure) was used to determine $L M N A$ gene structure. 
Sanger DNA sequencing. Variants in this family were determined via Sanger DNA sequencing. Primer Premier 5.0 software (Premier Biosoft India Pvt., Ltd., Indore, India) was applied to design primers for the targeted sequence. The gene sequence for LMNA (250 bp) was obtained from GenBank (https://www.ncbi.nlm.nih.gov/genbank/) (NM_170707.3) and was amplified using the following primers: Forward, 5'-ATGATCGCTTGGCGGTCTAC-3' and reverse, 5'-CGA ACTCACCGCGCTTTC-3'. The gene sequence for KCNQ4 (NM_004700.3) with a target sequence of $226 \mathrm{bp}$ according to the following primers: Forward, 5'-GGTAGGCTGGCT GTGATCTC-3' and reverse, 5'-GCAGGCCTCACCTTGCTA T-3'. The gene sequence for EYA transcriptional coactivator and phosphatase 1 (EYAl) was obtained from GenBank (NM_000503.5) and its target sequence was $250 \mathrm{bp}$ with the following primers: Forward, 5'-TCACAGCAGAATAAT GGCCAGT-3' and reverse, 5'-GTTTGGCAACTGGTGTAC GG-3'. The synthesized primers were obtained from Thermo Fisher Scientific. Inc. The PCR products were purified via an E.Z.N.A. ${ }^{\text {TM }}$ Gel Extraction kit (Omega Bio-Tek, Inc., Norcross, GA, USA) according to the manufacturer's protocols. PCR products were sequenced by Thermo Fisher Scientific, Inc.

\section{Results}

$N G S$ analysis. A total of $522,349,503$ bases from a typical run were obtained and 490,137,344 bases were $>$ Q20 . There were 3,533,834 reads and 3,523,608 were mapped. The mean read length was $135 \mathrm{bp}$ on average; $98 \%$ of the reads were on target and the mean depth was 324.4X. Furthermore, for 503 homozygous single nucleotide variants (SNVs), 0 homozygous multiple nucleotide variations (MNPs), 31 homozygous insertions/deletions (INDELs), 642 heterozygous SNVs, 1 heterozygous MNP and 39 heterozygous INDELs were determined; the present study reported $\mathrm{SNVs} /$ Total $=0.948, \mathrm{INDELs} / \mathrm{Total}=0.058, \mathrm{Ti} / \mathrm{Tv}$ ratio $(\mathrm{SNVs})=2.562$, NCBI dbSNP database concordance $=0.952$ and Heterozygotes/Homozygotes $=1.275$.

Determination of suspected pathogenic mutations in the proband. Following the removal of mutations that had a MAF $>1 \%, 6$ out of 157 mutations were identified in the ClinVar database (https://www.ncbi.nlm.nih.gov/clinvar/) as presented in Table II.

The present study examined all exonic and intronic borders of a targeted NGS panel comprising all genes known to be associated with cardiac diseases. Common variants identified were: $c . * 107 \mathrm{~A}>\mathrm{G}$ at untranslated regions -3 of exon 3 in hemoglobin A2 (HBA2), c.1122+52T $>\mathrm{C}$ in ryanodine receptor $1(R Y R I)$ and synonymous mutation (p.Val12=) in adenosine deaminase $(A D A)$. No rare nonsynonymous variants were detected except for heterozygous c.244G $>$ A (p.E82K) in LMNA (NM_170707.3) (Fig. 2A and B), c.546C $>$ G (p.F182L) in KCNQ4 (NM_004700.3) (Fig. 2C and D) and c.1276G $>$ A (p.G426S) in EYAl (NM_172058.3) (Fig. 2E and F). Additionally, c.*107A $>$ G (NM_000517.4) was identified in the 3 'untranslated region of exon 3 in $H B A 2$. c. $1122+52 \mathrm{~T}>\mathrm{C}$ (NM_000540.2) was located in the intronic region of RYRI and a synonymous (p.Val12=; NM_000022.2) mutation was identified in $A D A$. These mutations were all identified in 

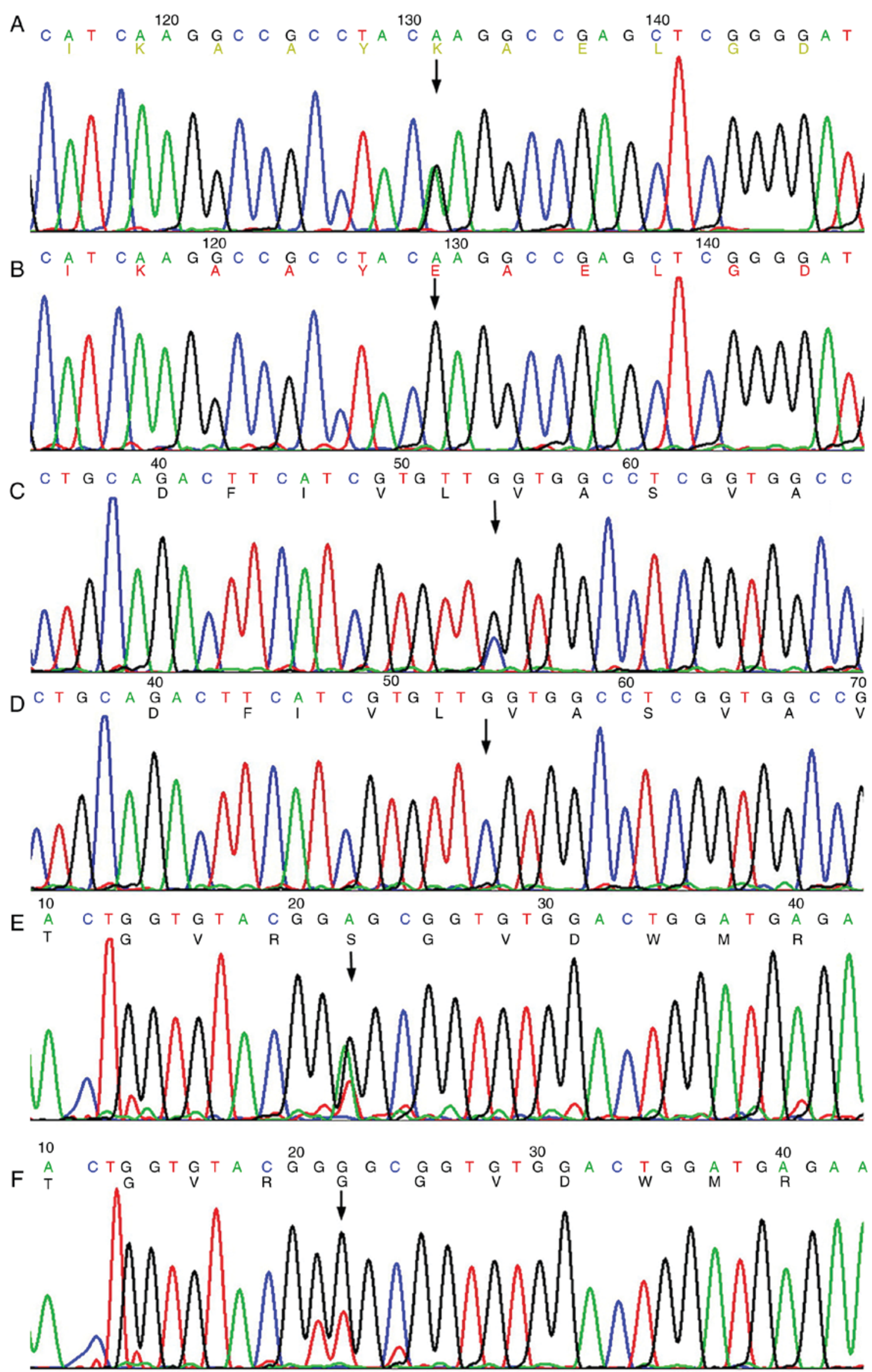

Figure 2. (A) Heterozygous p.E82K (c.244G>A) mutation in $L M N A$ was identified in the proband. (B) Sequencing of wild type $L M N A$. (C) Heterozygous p.F182L (c.546C>G) mutation in KCNQ4 identified in the proband. (D) sequencing of wild type KCNQ4. (E) Heterozygous p.G426S (c.1276G $>$ A) mutation in EYA1 was identified in the proband. (F) Sequencing of wild type EYA1. Arrows indicate mutation sites. LMNA, lamins A/C; KCNQ4, potassium voltage-gated channel subfamily KQT; EYA1, EYA transcriptional coactivator and phosphatase 1. 


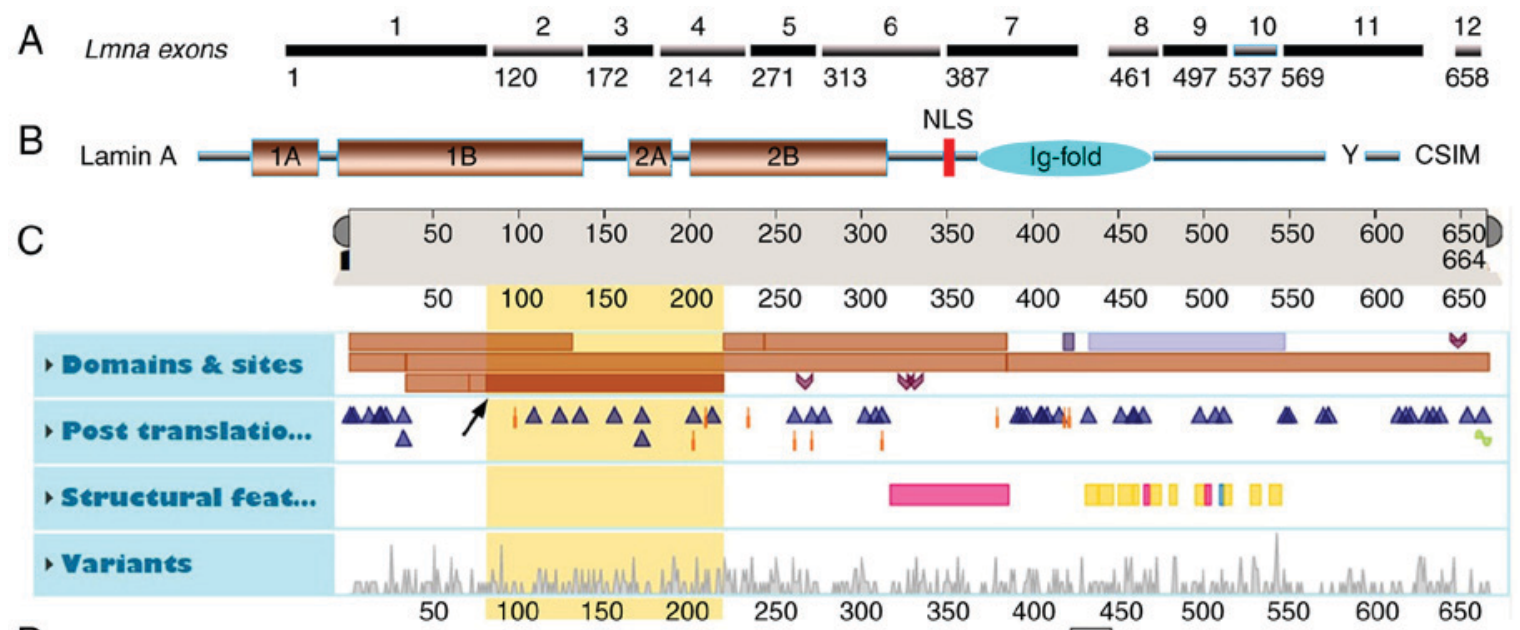

D Homo sapiens (Human)
Monodelphis domestica (Gray short-tailed opossum)

Figure 3. (A) Sketch map of exons 1-12 of LMNA. (B) Sketch map of lamin A protein. Y represents the site for zinc metallopeptidase STE24 hydrolysis and CSIM represents the target for farnesylation. (C) Features viewer and functional consequence of $L M N A$ p.E82K (GeneView). Yellow region of interest, amino acids 81-218; description of Coil 1B in LMNA and the arrow indicates the p.E82K mutation. (D) Homology comparison of lamin A protein among different species: Amino acid alignment revealed conservation among species for glutamic acid at position 82 in LMNA. CSIM, CaaX motif; LMNA, lamins A/C.

non-coding regions and may exhibit no effects on the onset of DCM.

These variants (p.E82K in LMNA, F182L in $K C N Q 4$ and G426S in EYAl) were absent in the database of 1000 genomes project (17). The NCBI ClinVar database revealed that the F182L mutation in $K C N Q 4$ has been reported to cause deafness $(18,19)$, and G426S in EYAl was reported as the pathogenic gene responsible for branchiootorenal syndrome 1 or Branchiootic syndrome 1 (20); similar results have been reported in the ExAC database (http://exac. broadinstitute.org/). The SIFT and PolyPhen-2 scores for p.F182L (c.546C $>\mathrm{G}$, rs80358273) of $K C N Q 4$ were 1.0 and 0.046, respectively; the SIFT and PolyPhen-2 scores for p.G426S (c.1276G>A, rs121909199) of EYAl were 0 and 0.891 , respectively. Finally, the SIFT and PolyPhen-2 scores for p.E82K (c.244G>A, rs59270054) of LMNA were 0 and 1, respectively.

Features viewer and functional consequence of LMNA p.E82K. LMNA p.E82K was located in the first exon of $L M N A$ and close to the $1 \mathrm{~B}$ structural domain margin in the rod area of the Lamin A/C N-terminus (Fig. 3A-C, yellow region of interest 81-218 and description of Coil 1B in LMNA; Table II). The analysis of $L M N A$ p.E82K across numerous species revealed that $L M N A$ Glu82 was conserved among all species examined with the exception of Rattus norvegicus (Rat) as presented in Fig. 3D.
DNA samples could not be obtained from the patients who had succumbed to heart disease-associated mortality; however, the proband (II-6) was identifiable in the present study. II-7 exhibited clinical features of DCM; the son (III-11) of II-5 (succumbed to mortality due to DCM), the son (III-7) of II-3 (succumbed to mortality due to heart disease), and the daughter (III-9) of II-4 (succumbed to mortality due to heart disease), along with III-13, -14, -11 and IV-7 were heterozygous for p.E82K in LMNA. However, no clinical data for DCM were obtained until the end of the present study. Furthermore, II- 1 and their daughters (III-2 and III-3), II-6 and their son (III-13) were heterozygous for p.F182L in $K C N Q 4$, while II-6 and III-13 were heterozygous for p.G426S in EYAl. Excluding a family member (III-3) who possessed the F182L mutation in $K C N Q 4$ and had slight hearing decline in the left ear as demonstrated via an audiometry examination, the hearing of II-6, II-1, III-2, and III-13, as well as that for the right ear of III-3 were all normal. The pedigree analysis demonstrated no family members with symptoms of branchiootorenal syndrome 1 or branchiootic syndrome 1 .

\section{Discussion}

LMNA is one of the most common pathogenic genes responsible FDC (3). At birth, no significant differences in the growth status between $L M N A$ knockout mice $\left(L M N A^{-/}\right)$and wild type mice were reported (16). After few weeks post-partum, 
knockout mice gradually exhibited severe growth delays, as well as muscle and heart atrophy; the $L M N A^{-/-}$mice were generated by Sullivan et al (21). Additionally, it has been demonstrated that these mice expressed a truncated lamin A product at the transcriptional and translational levels (22); however, the novel LMNA null mouse ( $\left.L M N A^{\mathrm{GT}-/}\right)$ created via gene trapping exhibited severe growth retardation and developmental defects of the heart, which resulted in mortality at 2-3 weeks post-partum without the occurrence of DCM or any notable progeroid phenotypes (23). At present, $>400$ mutations of LMNA have been identified, including deletions, missense mutations, insertions and nonsense mutations, which have been associated with a wide spectrum of diseases, including Emery-Dreifuss muscular dystrophy, accelerated aging disorders, such as the premature aging syndrome, Hutchinson-Gilford progeria, DCM, lipodystrophy and neurological disorders. These disorders have been classified as the 'laminopathies' $(24,25)$. Such conditions are defined as serious diseases resulting from mutations in genes encoding lamins, particularly $L M N A$, which encodes lamin A (12), or by mutations in genes that directly or indirectly encode lamin-associated proteins (26).

Mutations in LMNA mainly result in diseases, which feature nerve and muscle-associated symptoms with cardiac involvement; the first manifestations may include delayed electrical activities of the atrium and ventricle, followed by conduction system diseases, involving the sinoatrial node and atrioventricular node conduction disorders. Ventricular enlargement, heart failure, or sudden mortality may arise (24). Therefore, LMNA has been reported as the most common pathogenic gene for inhibiting FDC $(6,27)$. This may be associated with mutations in LMNA that cause abnormalities in expression and the localization of gap junction proteins (28). The rate of sudden mortality of patients with DCM resulting from mutations in $L M N A$ was reported to be higher compared with other causes of DCM9 (29). A 2005 meta-analysis of the clinical characteristics of 299 carriers of LMNA mutations revealed a sudden mortality rate of $46 \%$ (29). Furthermore, $>40$ mutations in $L M N A$ may lead to FDC with an abnormal conduction system as suggested by the ClinVar database. The majority of mutation sites were located in the 'rod' functional area of the lamin A/C proteins; via the 'rod'-like functional area and chromatin, the inner nuclear protein is connected as a whole, in which the two nucleolar proteins exhibit chain interactions, forming a coiled-coil dimer structure in the inner layer of the nuclear membrane, reported as the is $\alpha 2$ helix structure (30). Therefore, it may be speculated that a mutation in the 'rod'-like functional area may likely affect the $\alpha 2$ helix structure. Thus, the formation of pre-lamin A may occur and be unable to fulfil the functions of lamin A, which may lead to structural disorder of nuclear fibers.

The pathophysiological basis of cardiomyopathies caused by mutations in LMNA may involve abnormal signal transduction. Abnormal activation of extracellular signal-regulated kinase (ERK) and c-Jun N-terminal kinase, which belong to the mitogen-activated protein kinases (MAPK) family, can affect intracellular signaling. ERK and MAPK were pharmacologically inhibited in $L M N A$ mutant mice to prevent left ventricular dilatation and myocardial contractility $(31,32)$. In addition, it may be that lamins $\mathrm{A} / \mathrm{C}$ interact with transcription factors associated with transcriptional regulation (28). Lamins aid the sequestration of heterochromatin at the nuclear envelope; mutations in LMNA can also cause an increase in heterochromatin, thereby affecting gene expression, DNA self-repair and increased sensitivity to DNA damage (33).

At present, only three families with DCM resulting from similar mutations have been identified; the detection of the responsible mutation sites in the investigated family of the present study supports the hypothesis that $\mathrm{p} . \mathrm{E} 82 \mathrm{~K}$ in LMNA may exhibit strong pathogenic effects that results in DCM (34-36). Additionally, studies with larger populations have not been conducted; however, functional studies in vitro have indicated a significant effect on protein function. For example, mice exhibiting LMNA p.E82K demonstrated significantly suppressed Cx43 expression levels as well as cell cycle arrest at the $\mathrm{G} 0 / \mathrm{G} 1$ phase, and presented with clinical features of DCM $(28,37,38)$. It was also reported that the p.E82K mutation in LMNA may result in Charcot-Marie-Tooth hereditary neuropathy type 2 , an axonal (non-demyelinating) peripheral neuropathy characterized by heart weakness and atrophy, mild sensory loss, and normal or almost-normal nerve conduction velocities (https://www.ncbi.nlm.nih. gov/clinvar/variation/66882/\#clinical-assertions). This variant also segregated with the disease in the family with DCM in the present study. The other two pathogenic variants (p.F182L in KCNQ4 and p.G426S in EYAl) appeared to have no effects on this family with DCM; branchiootorenal syndrome 1 or branchiootic syndrome 1 was not observed in the present study. The phenotype and these two mutations did not segregate, suggesting that variation-associated information may not be effective to investigate pathological and quantitative risk assessments; these problems present a great challenge for accurate diagnosis (39). In summary, the p.E82K variant of $L M N A$ was deemed to be pathogenic based upon segregation studies, its absence in controls and functional evidence, as demonstrated by a systematic approach in assessing the clinical significance of genetic variants (40).

The underlying mechanism by which the LMNA p.E82K variant results in DCM remains unknown. In 2010, Sun et al (28) demonstrated that connexin $43(\mathrm{Cx} 43)$ protein expression was reduced by $40 \%$ in cells transfected with LMNA p.E82K compared with in cells transfected with wild type (WT) LMNA cDNA. Confocal imaging demonstrated that Cx43 localized to the cell interior via LMNA p.E82K, whereas WT LMNA localized to gap junctions, which mediates electrochemical connections between cells and determines the velocity of cardiac conduction (28). Conduction abnormalities caused by abnormal distributions and defects in lamins $\mathrm{A} / \mathrm{C}$ may lead to the occurrence of reentry arrhythmias, such as ventricular fibrillation (41). C-Jun N-terminal kinase and ERK1/2 associated with MAPK signaling were reported to regulate $\mathrm{Cx} 43$ protein expression $(42,43)$.

Suppressing the activity of ERKs can prevent damage to the heart due to mutations in LMNA (44). LMNA p.E82K can result in errors in the topology of lamins $\mathrm{A} / \mathrm{C}$ and its interacting protein emerin, which can damage cell membranes (45). The fragility of cell membranes can lead to abnormal chromatin condensation, which may be another mechanism underlying the onset of DCM induced by LMNA p.E82K (45). Cells 
transfected with $L M N A$ p.E82K were arrested at the $\mathrm{G}_{0} / \mathrm{G}_{1}$ phase, whereas cell groups without the mutation arrested at the $\mathrm{G}_{2} / \mathrm{M}$ phase in the presence of $\mathrm{H}_{2} \mathrm{O}_{2}$ (37). In addition, $L M N A$ p.E82K may have a tendency to promote cell immature transformation (46). These experiments indicated that $L M N A$ p.E82K may promote cell apoptosis, increase DNA synthesis and facilitate robust cell division. Such characteristics may lead to an abnormal number of cardiac cells, decreased muscle contractile function and weakened cardiac ejection function, thus resulting in DCM $(45,46)$. In addition, FDC-associated mutations of $L M N A$ resulting in FDC have distinct clinical features, and $25 \%$ of $L M N A$ carriers do not experience any notable symptoms during childhood (47); however, the penetrance of mutant $L M N A$ within carriers increases with age, attaining a rate of almost $100 \%$ at 60 years of age (48). This was supported by the results of the present study, which revealed that patients exhibiting clinical symptoms of DCM belonged to the I and II generations, while the younger III and IV generation carriers of p.E82K did not yet exhibit typical clinical manifestations of DCM. Mutations in lamins A/C can lead to poor prognoses of DCM, as well as a high incidence of sudden cardiac mortality and severe heart failure (49).

The traditional definition of cardiomyopathy, which was defined by the American Heart Association and the European Society of Cardiology, did not account for the genetic basis of cardiomyopathy (14). It was not until 2013 that a descriptive genotype-phenotype nosology system, MOGE (S), was proposed by the World Heart Federation, increasing awareness for the genetic etiology of DCM. MOGE(S) may serve as a comprehensive diagnostic, management and treatment tool for myocardial diseases, similar to the tumor node metastasis classification system for malignancy (50).

Collectively, the p.E82K mutation in LMNA may contribute to the pathogenesis of FDC with concomitant atrioventricular block as reported in the family investigated in the present study. The detection of the responsible mutation sites in the investigated family supports the hypothesis that $L M N A$ p.E82K may exhibit a strong pathogenic effect on DCM. Considering that the p.E82K mutation can cause a severe DCM clinical phenotype, this mutation may represent a therapeutic target or biomarker for the future diagnosis and or prognosis of DCM.

\section{Acknowledgements}

The authors would like to thank all participants in the present study for their cooperation.

\section{Funding}

The present study was supported by the Science and Technology Program of Fujian (grant nos. 2018Y0012, 2016J01501 and 2016Y0012) and the Financial Scheme for Young Talents Training Programme of Fujian Health Industry (grant no. 2015-ZQN-ZD-7).

\section{Availability of data and materials}

All data generated or analyzed during this study are included in this published article.

\section{Authors' contributions}

JWL conceived and designed the experiments. XFL, JWL, GL, YBZ and ZJ wrote the manuscript and performed the experiments. JWL, GL, XL and YBZ analyzed and interpreted the data. JWL and GL were responsible for critical revision of the content. All authors read and approved the final manuscript.

\section{Ethics approval and consent to participate}

The present study was performed in accordance with the Declaration of Helsinki and was approved by the Ethics Committee of Fujian Provincial Hospital, (Fuzhou, China). All participants and legal guardians of minors included in the study provided written informed consent.

\section{Patient consent for publication}

Consent for publication was obtained from all subjects involved in the present study.

\section{Competing interests}

The authors declare that they have no competing interests.

\section{References}

1. Hershberger RE and Siegfried JD: Update 2011: Clinical and genetic issues in familial dilated cardiomyopathy. J Am Coll Cardiol 57: 1641-1649, 2011.

2. Hershberger RE, Hedges DJ and Morales A: Dilated cardiomyopathy: The complexity of a diverse genetic architecture. Nat Rev Cardiol 10: 531-547, 2013

3. Zahr HC and Jaalouk DE: Exploring the Crosstalk Between LMNA and Splicing Machinery Gene Mutations in Dilated Cardiomyopathy. Front Genet 9: 231, 2018.

4. Skrzynia C, Berg JS, Willis MS and Jensen BC: Genetics and heart failure: A concise guide for the clinician. Curr Cardiol Rev 11: 10-17, 2015.

5. Yang J, Xu WW and Hu SJ: Heart failure: advanced development in genetics and epigenetics. Biomed Res Int 2015: 352734, 2015.

6. Arbustini E, Pilotto A, Repetto A, Grasso M, Negri A, Diegoli M, Campana C, Scelsi L, Baldini E, Gavazzi A and Tavazzi L: Autosomal dominant dilated cardiomyopathy with atrioventricular block: A lamin A/C defect-related disease: J Am Coll Cardiol 39: 981-990, 2002.

7. van Tintelen JP, Hofstra RM, Katerberg H, Rossenbacker T, Wiesfeld AC, du Marchie Sarvaas GJ, Wilde AA, van Langen IM, Nannenberg EA, van der Kooi AJ, et al: High yield of LMNA mutations in patients with dilated cardiomyopathy and/or conduction disease referred to cardiogenetics outpatient clinics. Am Heart J 154: 1130-1139, 2007.

8. van Spaendonck-Zwarts KY, van Rijsingen IA, van den Berg MP, Lekanne Deprez RH, Post JG, van Mil AM, Asselbergs FW, Christiaans I, van Langen IM, Wilde AA, et al: Genetic analysis in 418 index patients with idiopathic dilated cardiomyopathy: Overview of 10 years' experience. Eur J Heart Fail 15: 628-636, 2013.

9. Hutchison CJ and Worman HJ: A-type lamins: guardians of the soma? Nat Cell Biol 6: 1062-1067, 2004.

10. Scaffidi P and Misteli T: Lamin A-dependent nuclear defects in human aging. Science 312: 1059-1063, 2006.

11. Al-Haboubi T, Shumaker DK, Köser J, Wehnert M and Fahrenkrog B: Distinct association of the nuclear pore protein Nup153 with A- and B-type lamins. Nucleus 2: 500-509, 2011.

12. Worman HJ and Bonne G: 'Laminopathies': A wide spectrum of human diseases. Exp Cell 313: 2121-2133, 2007.

13. Freeman AR and Levine SA: Clinical significance of systolic murmurs: Study of 1000 consecutive 'noncardiac' cases. Ann Intern Med 6: 1371-1379, 1933. 
14. Mestroni L, Maisch B, McKenna WJ, Schwartz K, Charron P, Rocco C, Tesson F, Richter A, Wilke A and Komajda M: Guidelines for the study of familial dilated cardiomyopathies. Collaborative research group of the European human and capital. mobility project on familial dilated cardiomyopathy. Eur Heart J 20: 93-102, 1999.

15. AHA Medical/Scientific Statement: 1994 revisions to classification of functional capacity and objective assessment of patients with diseases of the heart. Circulation 90: 644-645, 1994.

16. Li Z, Huang J, Zhao J, Chen C, Wang H, Ding H, Wang DW and Wang DW: Rapid molecular genetic diagnosis of hypertrophic cardiomyopathy by semiconductor sequencing. J Transl Med 12: $173,2014$.

17. 1000 GenomesProject Consortium; Abecasis GR, Auton A, Brooks LD, DePristo MA, Durbin RM, Handsaker RE, Kang HM, Marth GT and McVean GA: An integrated map of genetic variation from 1,092 human genomes. Nature 491: 56-65, 2012.

18. Smith RJH and Hildebrand M: DFNA2 Nonsyndromic Hearing Loss. In: GeneReviews ${ }^{\circledR}$ [Internet]. Pagon RA, Adam MP, Ardinger HH, Wallace SE, Amemiya A, Bean LJH, Bird TD, Fong CT, Mefford HC, Smith RJH and Stephens K (eds). University of Washington, Seattle, WA, pp1993-2016, 2008.

19. Su CC, Yang JJ, Shieh JC, Su MC and Li SY: Identification of novel mutations in the KCNQ4 gene of patients with nonsyndromic deafness from Taiwan. Audiol Neurootol 12: 20-26, 2007.

20. Azuma N, Hirakiyama A, Inoue T, Asaka A and Yamada M: Mutations of a human homologue of the Drosophila eyes absent gene (EYA1) detected in patients with congenital cataracts and ocular anterior segment anomalies. Hum Mol Genet 9: 363-366, 2000.

21. Sullivan T, Escalante-Alcalde D, Bhatt H, Anver M, Bhat N, Nagashima K, Stewart CL and Burke B: Loss of A-type lamin expression compromises nuclear envelope integrity leading to muscular dystrophy. J Cell Biol 147: 913-920, 1999.

22. Jahn D, Schramm S, Schnölzer M, Heilmann CJ, de Koster CG, Schütz W, Benavente R and Alsheimer M: A truncated lamin A in the Lmna -/- mouse line: Implications for the understanding of laminopathies. Nucleus 3: 463-474, 2012.

23. Kubben N, Voncken JW, Konings G, van Weeghel M, van den Hoogenhof MM, Gijbels M, van Erk A, Schoonderwoerd K, van den Bosch B, Dahlmans V, et al: Post-natal myogenic and adipogenic developmental: Defects and metabolic impairment upon loss of A-type lamins. Nucleus 2: 195-207, 2011.

24. Kang SM, Yoon MH and Park BJ: Laminopathies; Mutations on single gene and various human genetic diseases. BMB Rep 51: 327-337, 2018

25. McNally EM, Golbus JR and Puckelwartz MJ: Genetic mutations and mechanisms in dilated cardiomyopathy. J Clin Invest 123: 19-26, 2013.

26. Worman HJ, Ostlund C and Wang Y: Diseases of the nuclear envelope. Cold Spring Harb Perspect Biol 2: a000760, 2010.

27. Bilińska ZT, Sylvius N, Grzybowski J, Fidziańska A, Michalak E, Walczak E, Walski M, Bieganowska K, Szymaniak E, Kuśmierczyk-Droszcz B, et al: Dilated cardiomyopathy caused by LMNA mutations. Clinical and morphological studies. Kardiol Pol 64: 812-821, 2006.

28. Sun LP, Wang L, Wang H, Zhang YH and Pu JL: Connexin 43 remodeling induced by LMNA gene mutation Glu82Lys in familial dilated cardiomyopathy with atrial ventricular block. Chin Med J (Engl) 123: 1058-1062, 2010.

29. van Berlo JH, de Voogt WG, van der Kooi AJ, van Tintelen JP, Bonne G, Yaou RB, Duboc D, Rossenbacker T, Heidbüchel H, de Visser M, et al: Meta-analysis of clinical characteristics of 299 carriers of LMNA gene mutations: do lamin A/C mutations portend a high risk of sudden death? J Mol Med (Berl) 83: 79-83, 2005.

30. Meune C, Van Berlo JH, Anselme F, Bonne G, Pinto YM and Duboc D: Primary prevention of sudden death in patients with lamin A/C gene mutations. N Engl J Med 354: 209-210, 2006.

31. Wu W, Shan J, Bonne G, Worman HJ and Muchir A: Pharmacological inhibition of c-Jun N-terminal kinase signaling prevents cardiomyopathy caused by mutation in LMNA gene. Biochim Biophys Acta 1802: 632-638, 2010.

32. Wu W, Muchir A, Shan J, Bonne G and Worman HJ: Mitogen-activated protein kinase inhibitors improve heart function and prevent fibrosis in cardiomyopathy caused by mutation in lamin A/C gene. Circulation 123: 53-61, 2011.
33. Mattout A,Pike BL, Towbin BD, Bank EM, Gonzalez-Sandoval A, Stadler MB, Meister P, Gruenbaum Y and Gasser SM: An EDMD mutation in $\mathrm{C}$. elegans lamin blocks muscle-specific gene relocation and compromises muscle integrity. Curr Biol 21: 1603-1614, 2011.

34. Novelli G and D'Apice MR: The strange case of the 'lumper' lamin $\mathrm{A} / \mathrm{C}$ gene and human premature ageing. Trends Mol Med 9: 370-375, 2003

35. Wang H, Zheng WY, Wang JZ, Wang XJ, Zhen YS, Song L, Zou YB and Hui RT: A novel LMNA gene mutation E82K associated with familial dilated cardiomyopathy. Zhonghua Xin Xue Guan Bing Za Zhi 33: 875-879, 2005 (In Chinese).

36. Wu X, Wang QK, Gui L, Liu M, Zhang X, Jin R, Li W, Yan L, Du R, Wang Q, et al: Identification of a new lamin A/C mutation in a Chinese family affected with atrioventricular block as the prominent phenotype. J Huazhong Univ Sci Technolog Med Sci 30: 103-107, 2010.

37. Wang H, Song XD, Wang SX, Fu CY, Sun K and Hui RT: Effects of a novel familial dilated cardiomyopathy associated LMNA gene mutation E82K on cell cycle of HEK293 cells. Zhonghua Xin Xue Guan Bing Za Zhi 35: 21-23, 2007 (In Chinese).

38. Lu D, Lian H, Zhang X, Shao H, Huang L, Qin C and Zhang L: LMNA E82K mutation activates FAS and mitochondrial pathways of apoptosis in heart tissue specific transgenic mice. PLoS One 5: e15167, 2010

39. Manrai AK, Ioannidis JP and Kohane IS: Clinical genomics: from pathogenicity claims to quantitative risk estimates. JAMA 315: 1233-1234, 2016

40. Duzkale H, Shen J, McLaughlin H, Alfares A, Kelly MA, Pugh TJ, Funke BH, Rehm HL and Lebo MS: A systematic approach to assessing the clinical significance of genetic variants. Clin Genet 84: 453-463, 2013.

41. Severs NJ, Bruce AF, Dupont E and Rothery S: Remodelling of gap junctions and connexin expression in diseased myocardium. Cardiovasc Res 80: 9-19, 2008.

42. Petrich BG, Eloff BC, Lerner DL, Kovacs A, Saffitz JE, Rosenbaum DS and Wang Y: Targeted activation of c-Jun $\mathrm{N}$-terminal kinase in vivo induces restrictive cardiomyopathy and conduction defects. J Biol Chem 279: 15330-15338, 2004.

43. Cho JH, Cho SD, Hu H, Kim SH, Lee SK, Lee YS and Kang KS: The roles of ERK1/2 and p38 MAP kinases in the preventive mechanisms of mushroom Phellinus linteus against the inhibition of gap junctional intercellular communication by hydrogen peroxide. Carcinogenesis 23: 1163-1169, 2002.

44. Muchir A, Shan J, Bonne G, Lehnart SE and Worman HJ Inhibition of extracellular signal-regulated kinase signaling to prevent cardiomyopathy caused by mutation in the gene encoding A-type lamins. Hum Mol Genet 18: 241-247, 2009.

45. Wang H, Wang J, Zheng W, Wang X, Wang S, Song L, Zou Y, Yao Y and Hui R: Mutation Glu82Lys in lamin A/C gene is associated with cardiomyopathy and conduction defect. Biochem Biophys Res Commun 344: 17-24, 2006.

46. Wang H, Zheng WY, Wang JZ, Song L, Zou YB, Zhen YS, Wang XJ and Hui RT: The effect of cell mitosis of LMNA gene mutation E82K associated with familial dilated cardiomyopathy. Mol Cardiol China 5: 777-779, 2005.

47. Sylvius N, Bilinska ZT, Veinot JP, Fidzianska A, Bolongo PM, Poon S, McKeown P, Davies RA, Chan KL, Tang AS, et al: In vivo and in vitro examination of the functional significances of novel lamin gene mutations in heart failure patients. J Med Genet 42: 639-647, 2005.

48. Malhotra R and Mason PK: Lamin A/C deficiency as a cause of familial dilated cardiomyopathy. Curr Opin Cardiol 24: 203-208, 2009.

49. Małek LA, Labib S, Mazurkiewicz L, Saj M, Płoski R, Tesson F and Bilińska ZT: A new c C > G, p.R541G lamin A/C mutation in a family with DCM and regional wall motion abnormalities (akinesis/dyskinesis): Genotype-phenotype correlation. J Hum Genet 56: 83-86, 2011.

50. Şahan E, Şahan S, Karamanlıŏlu M, Gul M and Tufekcioğlu O: The MOGE(S) classification: A TNM-like classification for cardiomyopathies. Herz 41: 503-506, 2016.

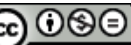

This work is licensed under a Creative Commons Attribution-NonCommercial-NoDerivatives 4.0 International (CC BY-NC-ND 4.0) License. 